\title{
Mixed-mode crack tip fields in a polycrystalline aluminum alloy
}

\author{
Marcel Wicke ${ }^{1,1}$, Angelika Brueckner-Foit ${ }^{1}$ \\ ${ }^{1}$ Institute for Materials Engineering, University of Kassel, D-34125 Kassel, Germany
}

\begin{abstract}
Carefully performed experiments with long cracks in the nearthreshold regime have shown that the crack tip field of these cracks significantly deviate from the expected mode-I butterfly-shaped ones and resemble strongly to mixed-mode crack tip fields. A simulation study using a crystal plasticity (CP) approach has been utilized in order to understand this phenomenon. To this end, a digital twin of an aluminum sample fatigued in the near-threshold regime was generated with the help of electron backscatter diffraction (EBSD) and X-ray tomography. Once set-up, the digital twin was loaded in uniaxial tension using the fast spectral solver implemented in the Düsseldorf Advanced Material Simulation Kit (DAMASK). The versatility of this experimental-computational approach for studying the strain partitioning at the crack tip is demonstrated in this work.
\end{abstract}

\section{Introduction}

Interest in the very high cycle fatigue (VHCF) regime is typically focused on the crack initiation phase, which is regarded as the life-determining phase. The importance of the crack propagation phase, however, may increase if cracks initiate from pre-existing defects. In that case, a notable amount of lifetime is spent in the crack propagation phase with long lifetimes being achieved in the case of near-threshold fatigue. However, very low stress amplitudes required for keeping the crack in the near-threshold regime are supposed to cause unexpected crack growth phenomenons. Similar effects as reported for small cracks, which are known to extend in an intermittent, shear-dominated pattern below the long-crack threshold (e.g. [13]), may occur under such circumstances making it worthwhile to include the effect of very small cyclic loads on crack extension in the VHCF research. Moreover, the shear-dominated parts of the crack path induce a mixed-mode situation at the crack tip under nominally normal external loading.

A test program was therefore set up in an authors' previous study [4] to analyze the nearthreshold crack extension mechanisms of long cracks in a commercial aluminum alloy used in peak-aged and overaged condition. Fatigue crack growth experiments were performed at nominally constant $\Delta \mathrm{K}$-values close to the threshold in a conventional resonant testing machine according to the procedure described in [4]. It was found that the crack tip field of these cracks strongly deviate from the typical fracture mechanics mode-I case. As it can be seen in Fig. 1 showing the crack tip field of a specimen taken from peak-aged material in

\footnotetext{
Corresponding author: marcel.wicke@,uni-kassel.de
} 
transverse direction as an example, the major part of the plastic deformation ahead of the crack tip coincided with the crack path direction. This type of crack tip field corresponds very much to a mixed-mode situation or, alternatively, a stage-I crack in which all dislocations are aligned on a slip system with high Schmid factor.

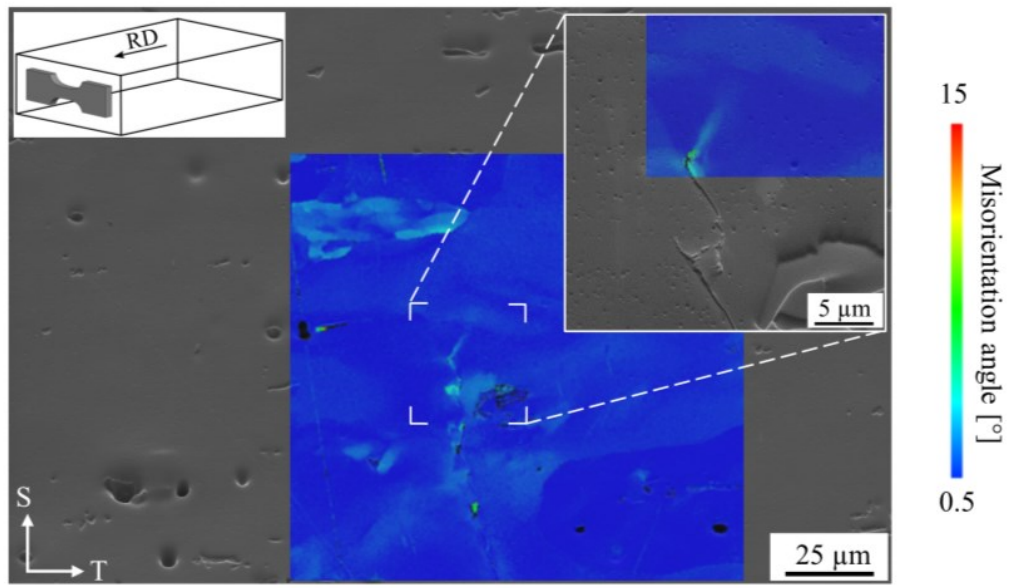

Fig. 1. Crack tip field of a peak-aged aluminum sample taken in transverse direction, which was tested at a load of $\Delta \mathrm{K}=1.4 \mathrm{MPa} \sqrt{\mathrm{m}}$.

Crystal plasticity (CP) simulations were performed in this work to understand the mixedmode type crack tip fields observed in the experiment. The basic hypothesis is that local mixed-mode loading can occur in the vicinity of the crack tip due to the anisotropy of the grain structure. This required to generate a digital twin of the fatigue tested samples, i.e. a three-dimensional digitized model of the experimentally characterized microstructure. While most studies concerned with the creation and evaluation of digital twins focus on capturing the grain structure (see e.g. [5,6]), electron backscatter diffraction (EBSD) and highresolution X-ray micro-computed tomography $(\mu-\mathrm{CT})$ were used here for getting a realistic three-dimensional representation of both the grain structure and microstructural features including the crack. Since the amount of analytical effort rises significantly with each additional detail required for defining the digital microstructural twin, special emphasis was put on including the important microstructural features around the crack tip but also on keeping the model as simple as possible at the same time.

The paper is structured as follows: The description of the modeling approach, namely the crystal plasticity model and the numerical solution strategy as implemented into DAMASK, in section 2 is followed by an overview of the simulation setup including details of the microstructure model. After presenting and discussing preliminary results in section 4, we give an outlook on further improvements of the employed approach.

\section{Modeling strategy}

The simulations presented in this work were performed using the multiphysics simulation package DAMASK [7]. A spectral solver, which allows a direct use and mapping of the experimentally obtained microstructure data, is employed to solve the mechanical boundary value problem for static mechanical equilibrium. The basic principles of the crystal plasticity formulation and the numerical solver are presented in the following. For further details, we refer to the recent work by Roters et al. [7]. 


\subsection{Crystal plasticity}

A conventional phenomenological crystal plasticity constitutive law $[8,9]$ for face-centered cubic (fcc) crystals was used to capture the behavior of the aluminum matrix. It is based on the assumption that plastic slip $\gamma$ occurs on a slip system $\alpha$ once the resolved shear stress $\tau^{\alpha}$ exceeds a critical value $\xi^{\alpha}$. The critical shear stress on each of the $12 \mathrm{fcc}$ slip systems is assumed to evolve from an initial value $\xi_{0}$ to a saturation value $\xi_{\infty}$ as a result of slip on all $\langle 011\rangle\{111\}$ systems $\beta=1, \ldots, 12$ according to the relationship

$$
\dot{\xi}^{\alpha}=h_{0}\left|\dot{\gamma}^{\beta}\right|\left|1-\frac{\xi^{\beta}}{\xi_{\infty}^{\beta}}\right|^{a} \operatorname{sgn}\left(1-\frac{\xi^{\beta}}{\xi_{\infty}^{\beta}}\right) h_{\alpha \beta}
$$

with the initial hardening $h_{0}$, interaction coefficients $h_{\alpha \beta}$ and a numerical parameter $a$. The shear rate on system $\alpha$ is then computed as

$$
\dot{\gamma}^{\alpha}=\dot{\gamma}_{0}\left|\frac{\tau^{\alpha}}{\xi^{\alpha}}\right|^{n} \operatorname{sgn}\left(\frac{\tau^{\alpha}}{\xi^{\alpha}}\right)
$$

with $n$ and $\dot{\gamma}_{0}$ being the inverse shear rate sensitivity and reference shear rate, respectively. The sum of the shear rates on all systems determines the plastic velocity gradient for the finite strain formulation used in DAMASK.

\subsection{Numerical Solver}

The simulations were conducted using the spectral formulation coupled to DAMASK, which was presented by Eisenlohr et al. [10] and recently extended by Shanthraj et al. [11]. Pioneered by Moulinec and Suquet [12], the spectral method approach based on the fast FOURIER transform has gained significant attention in computational material mechanics $[13,14]$. Good computational performance, in particular when simulating periodic microstructures, combined with the straightforward use of experimental data sets consisting of regularly arranged measurement points are the notable advantages of this method. One drawback of the early spectral method variants, namely the poor convergence rate for materials with a high stiffness or strength difference, has furthermore been overcome by replacing the original fix-point scheme by sophisticated root-finding algorithms $[11,15,16]$.

\section{Simulation setup}

The material examined in this study is the aluminum alloy EN AW-6082, which was peakaged and overaged at the TU Dresden. A rolled sheet with a thickness of $20 \mathrm{~mm}$ served as base material, its microstructure being defined by elongated grains with a maximum extension of up to $2000-3000 \mu \mathrm{m}$ in longitudinal (L) direction and maximum lengths of $400-500 \mu \mathrm{m}$ and $100-200 \mu \mathrm{m}$ in transverse (T) and short transverse (S) direction, respectively. Two types of primary precipitates with sizes up to $20-25 \mu \mathrm{m}$ can be detected: Mg-based ones $\left(\mathrm{Mg}_{2} \mathrm{Si}\right)$ and $\mathrm{Fe}$-based ones $(\mathrm{Al}(\mathrm{Fe}, \mathrm{Mn}) \mathrm{Si})$, which are arranged in a line-like fashion in rolling direction (RD). This effect is less pronounced in the case of the $\mathrm{Mg}_{2} \mathrm{Si}$ precipitates.

Flat dog-bone specimens with a total length of $40 \mathrm{~mm}$ were machined out of the sheet material and were fatigued in the near-threshold regime according to the procedure described in [4]. In the following it is outlined how a microstructural model was created from the fatigued specimen shown in Fig. 1. Moreover, the parameters for the constitutive model and the loading boundary conditions are given. 


\subsection{Microstructural characterization}

EBSD and laboratory-based $\mu$-CT were employed for obtaining a three-dimensional microstructure data set of the fatigued sample. To allow three-dimensional imaging at a spatial resolution appropriate for visualizing the fatigue crack, a cylindrical sample with a diameter of about $0.65 \mu \mathrm{m}$ containing the fatigue crack was prepared out of the flat dog-bone specimen using wire electro-discharge machining.

Following sample preparation, the specimen was analyzed in a scanning electron microscope (SEM, ZEISS Ultra 55) equipped with a Bruker e-Flash ${ }^{\mathrm{HR}}$ EBSD detector to capture to grain structure (Fig. 2a). EBSD data were acquired using an aperture of $120 \mu \mathrm{m}$ at an accelerating voltage of $20 \mathrm{kV}$ and a working distance of about $16 \mathrm{~mm}$. Esprit 2.1 (Bruker Nano) was used for data acquisition and indexing, while post-processing of the EBSD data set was done using DREAM.3D [17] software.
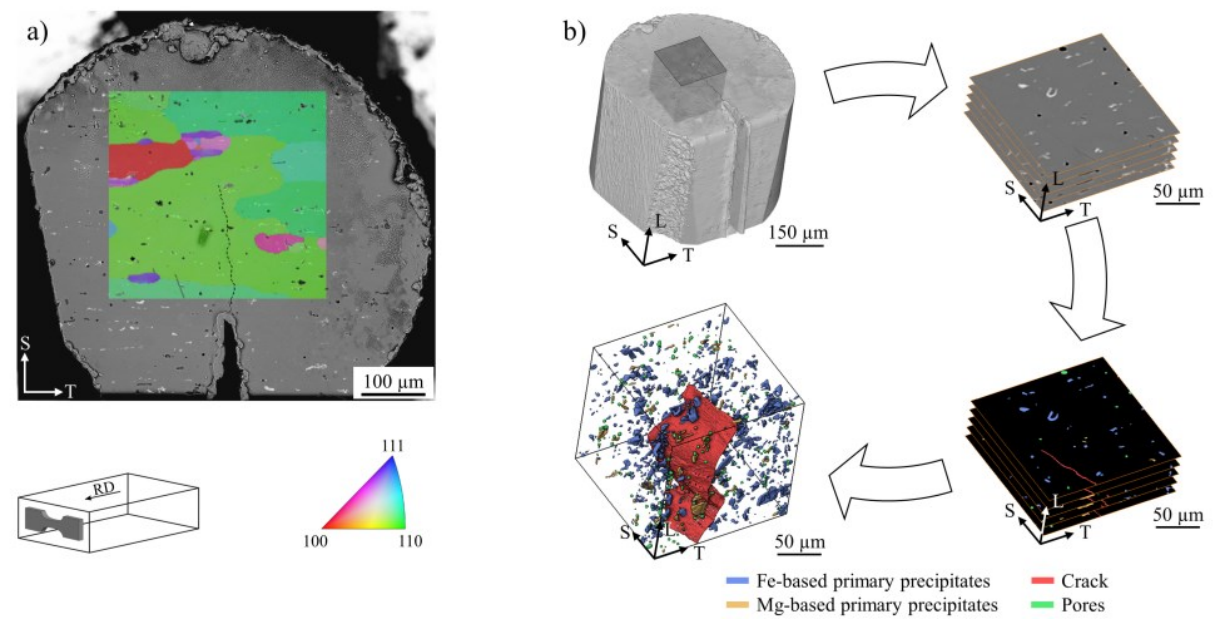

Fig. 2. Data sets obtained by a) EBSD and b) $\mu$-CT characterization of the fatigued sample. Colors of the orientation map shown in a) according to inverse pole figure parallel to longitudinal direction.

The three-dimensional measurement was carried out at $4 \mathrm{X}$ optical magnification with a ZEISS Xradia 520 Versa X-ray microscope at a voltage of $40 \mathrm{kV}$ and a power of $3 \mathrm{~W}$. The cylindrical sample was placed between the X-ray source and the $2 \mathrm{k} \times 2 \mathrm{k}$ detector with a source-to-sample and source-to-detector distance of $10.4 \mathrm{~mm}$ and $100 \mathrm{~mm}$, respectively. With the detector set to a $2 \times 2$ pixel binning, this provided an effective pixel size of $0.7 \mu \mathrm{m}$ similar to previous studies focusing on the successful imaging of fatigue cracks using synchrotron radiation (e.g. [18-20]). 3201 radiographs were collected per $360^{\circ}$ sample rotation with an exposure time of $45 \mathrm{~s}$ per radiograph to obtain a high signal-to-noise ratio.

Reconstruction of the raw transmission images was done using the ZEISS XMReconstructor software package employing a filtered back-projection algorithm. Avizo 9.2 (VSG) was used to perform image analysis including filtering and segmentation. The procedure is schematically illustrated in Fig. 2b. A region of interest (ROI) was first extracted from the reconstructed data set by cropping a volume element (VE) of $256^{3}$ voxels, corresponding to about $180 \times 180 \times 180 \mu \mathrm{m}^{3}$ (dark cube in Fig. 2b, top left). To reduce the noise level, a non-local means filter was applied to the reconstructed slices of the VE afterwards (Fig. 2b, top right). The filtered images were then processed using simple thresholding to segment the crack, primary precipitates and pores (Fig. 2b, bottom right). Inaccuracies, occurring particularly very close to the crack front cause of resolution limits, were subsequently corrected manually to obtain the final label field (Fig. 2b, bottom left). 


\subsection{Model creation}

The model required for conducting the $\mathrm{CP}$ simulations can be directly created from the experimentally characterized microstructure. To this end, the label field obtained by the segmentation (Fig. 2b, bottom left) was exported in ASCII format. Python code was used to convert the data set into the geometric description readable by DAMASK, which was discretized by a regular voxel grid with $64 \times 64 \times 64$ grid points. As illustrated in Fig. 3, the measured grain structure was subsequently mapped onto the surface of the spectral solver description and the grains were expanded in-depth, i.e. the grains were simplified as columns. This kind of simplification was justified for a first principle study since the size of the VE was small compared to the grain extension in rolling direction. All grid points belonging to a grain were assigned the same crystal orientation obtained by grain segmentation in DREAM.3D [17]. A layer of soft dilatational material, akin to air, of 6 voxel thickness was attached on top of the polycrystalline structure resulting in boundary conditions that are reminiscent of a free surface.

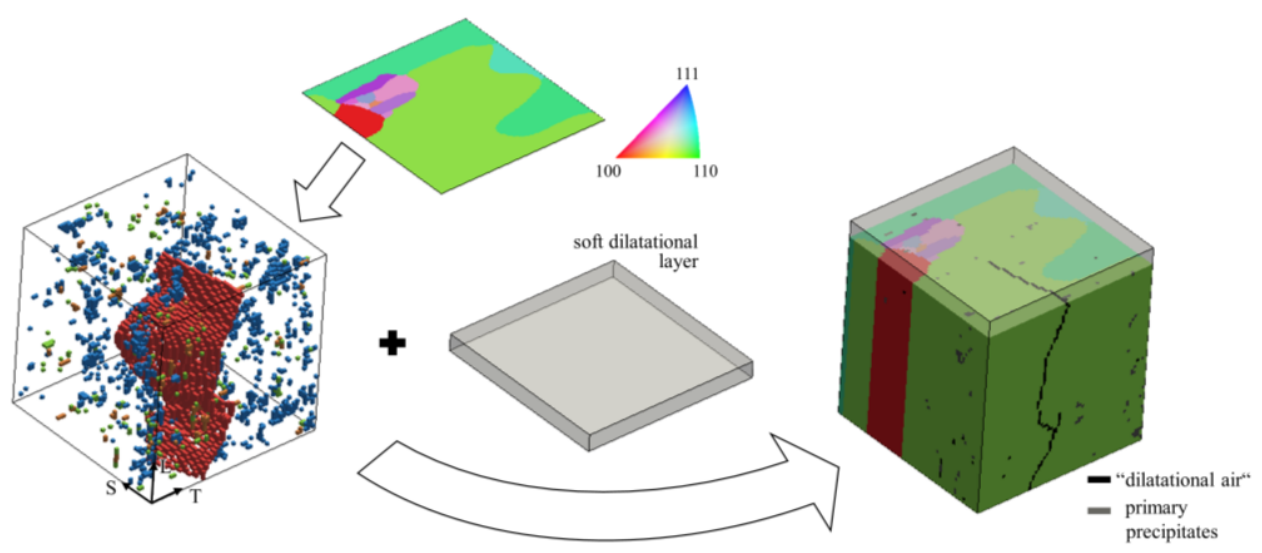

Fig. 3. Creation of microstructural model readable by DAMASK.

The parameters used to model the constitutive response of the aluminum fcc crystal and microstructural features are given in Table 1 .

Table 1. Material properties for aluminum matrix, primary precipitates, and dilatational air. Note that $h_{\alpha \beta}$ is used only in the CP model and Taylor factor $M$ only in the isotropic dilatational model.

\begin{tabular}{lllll}
\hline Property & Value & & Unit \\
\cline { 2 - 4 } & Aluminum matrix & Primary Precipitates & Dilatational air & \\
\hline$C_{11}$ & $106.8 \times 10^{9}$ & $237.53 \times 10^{9}$ & $10.0 \times 10^{9}$ & $\mathrm{~Pa}$ \\
$C_{12}$ & $60.4 \times 10^{9}$ & $70.83 \times 10^{9}$ & $0.0 \times 10^{9}$ & $\mathrm{~Pa}$ \\
$C_{44}$ & $28.3 \times 10^{9}$ & & & $\mathrm{~Pa}$ \\
\hline$\dot{\gamma}_{0}$ & $1.0 \times 10^{-3}$ & & $1.0 \times 10^{-3}$ & $\mathrm{~s}^{-1}$ \\
$\tau_{0}$ & $31.0 \times 10^{6}$ & & $0.3 \times 10^{6}$ & $\mathrm{~Pa}$ \\
$\tau_{\infty}$ & $63.0 \times 10^{6}$ & $0.6 \times 10^{6}$ & $\mathrm{~Pa}$ \\
$h_{0}$ & $75.0 \times 10^{6}$ & $1.0 \times 10^{6}$ & $\mathrm{~Pa}$ \\
$a$ & 2.25 & 2.0 & \\
$n$ & 20.0 & 5.0 & \\
Coplanar $h_{\alpha \beta}$ & 1.0 & & & \\
Non-coplanar $h_{\alpha \beta}$ & 1.4 & 3.0 & \\
$M$ & & & & \\
\hline
\end{tabular}


Since obtaining suitable parameters for the employed phenomenological crystal plasticity model $[21,22]$ is a challenging task by itself, an existing data set reproducing the behavior of a soft aluminum alloy [10] was used to describe the matrix material. Similar to the layer on top of the polycrystalline structure, the void volumes, i.e. the crack and pores that cannot be taken out of the computation domain as in e.g. finite element simulations, were modeled as soft dilatational and low strength materials ("dilatational air" in Table 1) using the constitutive description proposed by Maiti and Eisenlohr [23]. Isotropic elastic behavior was assumed inside the primary precipitates for the sake of simplicity. The elastic stiffness values were chosen based on a recent study by $\mathrm{Ma}$ [24] without distinguishing between $\mathrm{Fe}$ - and $\mathrm{Mg}$ based precipitates.

Uniaxial tension loading along the transverse direction was realized by enforcing the average deformation gradient rate tensor and the complementary average first PIOLAKIRCHHOFF tensor to be

$$
\dot{\overline{\mathbf{F}}}=\left[\begin{array}{ccc}
10^{-3} & 0 & 0 \\
0 & * & 0 \\
0 & 0 & *
\end{array}\right] \mathrm{s}^{-1} \text { and } \quad \overline{\mathbf{P}}=\left[\begin{array}{ccc}
* & * & * \\
* & 0 & * \\
* & * & 0
\end{array}\right] \mathrm{Pa}
$$

with "*” indicating components for which complementary boundary conditions are specified. These conditions were applied for $40 \mathrm{~s}$ in 400 uniform time increments, thus reaching a final strain of 0.04. It should be noted that the values in Eq. (3) are prescribed as volume average since the microstructure model was periodically repeated as it is characteristic for the spectral solution method.

\section{Results}

Fig. 4 shows the spatial distribution of the equivalent (VON MISES) strain $\varepsilon_{\mathrm{VM}}$ at $\varepsilon=0.010$, 0.025 , and 0.040 with $\varepsilon$ being the logarithmic (true) strain along loading direction. A strong strain partitioning is observed ahead of the crack tip. Instead of developing the classical mode-I butterfly-shaped crack tip field, the strain is localized in a band oriented at about $45^{\circ}$ with respect to the loading direction very similar to the findings in the experiment. This implies that the observed anomaly in the crack tip field can be traced back to the mixed-mode loading caused by grain anisotropy
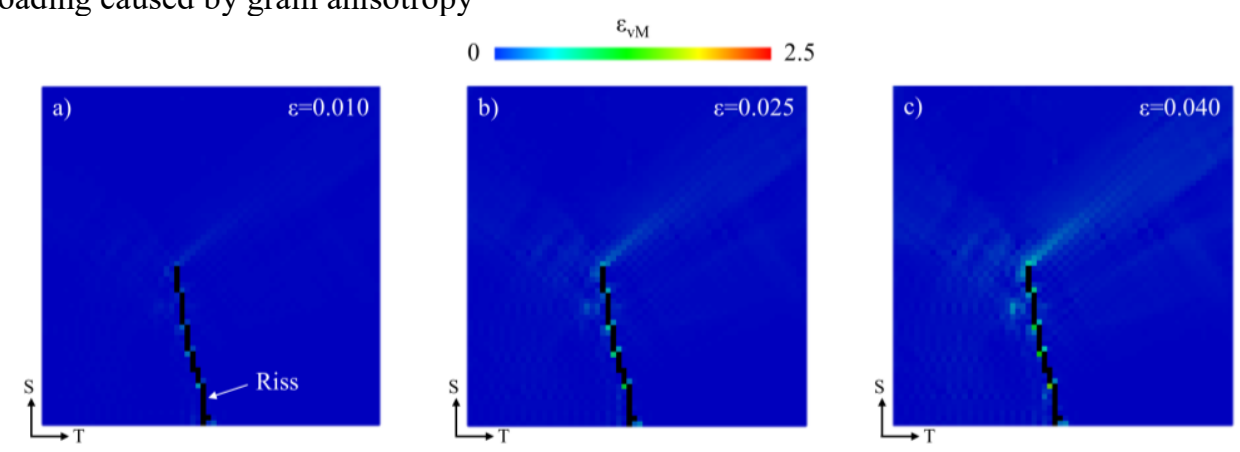

Fig. 4. Equivalent strain $\varepsilon_{\mathrm{vM}}$ at increasing strain level $\varepsilon$ mapped onto the undeformed microstructure.

The digital twin is suitable to describe the overall strain distribution trends observed in the experiment correctly. However, there are some differences regarding the exact location of the highly strained zone ahead of the crack tip. These deviations may most likely be attributed to limitations of the experimental approach used for creating the digital twin. Since SEM is a surface analysis technique, it cannot be used to reveal three-dimensional data sets of the investigated microstructure. A columnar grain structure has hence inevitably to be introduced in the simulation model. Differences in the local deformation behavior are thus 
expected particularly when taking into account the additional simplifications in the underlying simulation methodology, i.e. the usage of an existing parameter set to model the constitutive response of the aluminum fcc crystal. A higher resolved microstructure discretization is furthermore required to describe the crack morphology more accurately in the digital twin, which however would cause a dramatic increase in computational cost.

It can be concluded that the experimental-numerical methodology presented here is able to capture the plastic zone ahead of the crack tip in a realistic way. Further authenticity can be implemented, and an improved agreement between simulation and experiments can most likely be achieved when a higher resolved microstructure discretization is used and if the three-dimensional grain structure could be successfully taken into account.

\section{Conclusion and outlook}

A digital twin of a fatigued aluminum sample based directly on data sets obtained by EBSD and $\mu$-CT characterization has been utilized for a crystal plasticity simulation study using a fast spectral solver implemented in DAMASK. The three-dimensional model, by including details such as crystallographic orientation, crack morphology and distribution of the microstructural features, allows to analyze the strain partitioning at the crack tip. In agreement with the experimental observations, the simulated crack tip field deviates from the typical fracture mechanics mode-I case and resembles to a mixed-mode crack tip field. The simulation shows that this effect can be traced back to the multiaxial stress field induced by grain anisotropy and is thus fundamentally different from the single slip line associated with stage-I mircocracks.

The modeling method combined with the approach for obtaining a digital twin by EBSD and $\mu-\mathrm{CT}$ is hence a promising route for efficiently studying the strain partitioning at the crack tip. Additional microstructural authenticity, however, is required to get a more in-depth understanding of the crack tip field deviations. A realistic three-dimensional representation of the grain structure will therefore be included in an upcoming study using a grain boundary wetting with liquid gallium. Moreover, further authenticity will be introduced in the microstructure model by extracting the phase properties of the aluminum matrix from the microstructure itself using "virtual single crystal" experiments based on nano-indentation according to the procedure developed by Zambaldi and Raabe [25] and Zambaldi et al. [21].

The authors would like to thank Martin Diehl, Max-Planck-Institut für Eisenforschung GmbH, Germany, for his helpful suggestions. Financial support from the German Research Foundation (DFG) is gratefully acknowledged.

\section{References}

1. E.R. De Los Rios, H.J. Mohamed, K.J. Miller. A micro-mechanics analysis for short fatigue crack growth. Fatigue Fract Eng Mater Struct, 8: 49-64 (1985)

2. A. Navarro, E.R. De Los Rios. Short and long fatigue crack growth: a unified model. Fatigue Fract Eng Mater Struct, 11: 383-96 (1988)

3. K. Hussain, E. R. De Los Rios, A. Navarro. A two-stage micromechanics model for short fatigue cracks. Eng Fract Mech, 44: 425-36 (1993)

4. M. Wicke, A. Brueckner-Foit, T. Kirsten, M. Zimmermann, F. Buelbuel, H.J. Christ. Near-Threshold Crack Extension Mechanism in an Aluminum Alloy Studied by SEM and X-Ray Tomography. Int J Fatigue, 119: 102-11 (2019)

5. M. Diehl, D. An, P. Shanthraj, S. Zaefferer, F. Roters, D. Raabe. Crystal Plasticity Study in Stress and Strain Partitioning in a Measured 3D Dual Phase Steel Microstructure. Phys Mesomech, 20: 311-23 (2017) 
6. Z. Zhao, M. Ramesh, D. Raabe, A.M. Cuitino, R. Radovitzky. Investigation of threedimensional aspects of grain-scale plastic surface deformation of an aluminum oligocrystal. Int J Plast, 24: 2278-97 (2008)

7. F. Roters, M. Diehl, P. Shanthraj, P. Eisenlohr, C. Reuber, S.L. Wong, T. Maiti, A. Ebrahimi, T. Hochrainer, H.-O. Fabritius, S. Nikolov, M. Friák, N. Fujita, N. Grilli, K.G.F. Janssens, N. Jia, P.J.J. Kok, D. Ma, F. Meier, E. Werner, M. Stricker, D. Weygand, D. Raabe. DAMASK - The Düsseldorf Advanced Material Simulation Kit for modeling multi-physics crystal plasticity, thermal, and damage phenomena from the single crystal up to the component scale. Comput Mater Sci, 158: 420-78 (2019)

8. J.W. Hutchinson. Bounds and self-consistent estimates for creep of polycrystalline materials. Proc R Soc A: Math, Phys Eng Sci, 348: 101-27 (1976)

9. D. Peirce, R.J. Asaro, A. Needleman. Material rate dependence and localized deformation in crystalline solids. Acta Metall, 31: 1951-76 (1983)

10. P. Eisenlohr, M. Diehl, R.A. Lebensohn, F. Roters. A spectral method solution to crystal elasto-viscoplasticity at finite strains. Int J Plast, 46: 37-53 (2013)

11. P. Shanthraj, P. Eisenlohr, M. Diehl, F. Roters. Numerically robust spectral methods for crystal plasticity simulations of heterogeneous materials. Int J Plast, 66: 31-45 (2015)

12. H. Moulinec P. Suquet. A numerical method for computing the overall response of nonlinear composites with complex microstructure. Comp Meth Appl Mech Eng, 157: 69-94 (1998)

13. S.B. Lee, R.A. Lebensohn, A.D. Rollett. Modeling the viscoplastic micromechanical response of two-phase materials using Fast Fourier Transforms. Int J Plast, 27: 707-27 (2011)

14. J. Sliseris, H. Andrä, M. Kabel, B. Dix, B. Plinke, O. Wirjadi, G. Frolovs. Numerical prediction of the stiffness and strength of medium density fiberboards. Mech Mater, 79: 73-84 (2014)

15. J. Zeman, J. Vondřejc, J. Novák, I. Marek. Accelerating a FFT-based solver for numerical homogenization of periodic media by conjugate gradients. J Comp Phys, 229: 8065-71 (2010)

16. L. Gélébart, R. Mondon-Cancel. Non-linear extension of FFT-based methods accelerated by conjugate gradients to evaluate the mechanical behavior of composite materials. Comput Mater Sci, 77: 430-39 (2013)

17. M.A. Groeber, M.A. Jackson. DREAM. 3D: a digital representation environment for the analysis of microstructure in 3D. Integ Mater Manuf Innov, 3: 5 (2014)

18. T.J. Marrow, J.-Y. Buffière, P.J. Withers, G. Johnson, D. Engelberg. High resolution Xray tomography of short fatigue crack nucleation in austempered ductile cast iron. Int $\mathrm{J}$ Fatigue, 26: 717-25 (2004)

19. J.-Y. Buffière, E. Ferrie, H. Proudhon, W. Ludwig. Three-dimensional visualisation of fatigue cracks in metals using high resolution synchrotron X-ray micro-tomography. Mater Sci Technol, 22: 1019-24 (2006)

20. M. Herbig, A. King, P. Reischig, H. Proudhon, E.M. Lauridsen, J. Marrow, J.-Y. Buffière, W. Ludwig. 3-D growth of a short fatigue crack within a polycrystalline microstructure studied using combined diffraction and phase-contrast X-ray tomography. Acta Mater, 59: 590-601 (2011)

21. C. Zambaldi, Y. Yang, T.R. Bieler, D. Raabe. Orientation informed nanoindentation of $\alpha$-titanium: Indentation pileup in hexagonal metals deforming by prismatic slip. J Mater Res, 27: 356-67 (2012).

22. C.C. Tasan, M. Diehl, D. Yan, C. Zambaldi, P. Shanthraj, F. Roters, D. Raabe. Integrated experimental-numerical analysis of stress and strain partitioning in multi-phase alloys. Acta Mater, 81: 386-400 (2014) 
23. T. Maiti, P. Eisenlohr. Fourier-based spectral method solution to finite strain crystal plasticity with free surfaces. Scri Mater, 145: 37-40 (2018)

24. D. Ma. Elastic properties of Mn-rich $\alpha$ intermetallic phase in engineering aluminum alloys: An ab initio study. J Appl Phys, 124: 085109 (2018)

25. C. Zambaldi, D. Raabe. Plastic anisotropy of $\gamma$-TiAl revealed by axisymmetric indentation. Acta Mater, 58: 3516-30 (2010) 